\title{
Obstacle Avoidance Strategy Based on Adaptive Potential Fields Generated by an Electronic Stick
}

\author{
Ernesto P. Lopes \\ Institute of Mathematics - COPPE \\ Federal University of Rio de Janeiro \\ P.O. Box 2324, Rio de Janeiro, RJ 20001-970, Brazil \\ lopes@cos.ufrj.br
}

\author{
Eliana P.L. Aude, Julio T.C. Silveira, Henrique Serdeira \\ Computer Center - NCE \\ Federal University of Rio de Janeiro \\ P.O. Box 2324, Rio de Janeiro, RJ 20001-970, Brazil \\ elaude@nce.ufrj.br
}

\begin{abstract}
In our previous work, an obstacle avoidance algorithm, which used potential fields and a similar strategy to that adopted by a blind person to avoid obstacles whilst walking, was proposed. The problem analyzed consists of an AGV (Autonomous Guided Vehicle) which moves within an office environment with a known floor plan and uses an "electronic stick" made up of infrared sensors to detect unknown obstacles in its path. Initially, a global potential navigation function, defined for each room in the floor plan, incorporates information about the dimensions of the room and the position of the door which the AGV must use to leave the room. Whilst the AGV moves, this global potential navigation function is properly modified to incorporate information about any newly detected obstacle. The main interesting aspect of the proposed approach is that the potential function adaptation involves very low computational burden allowing for the use of Ultra-fast AGVs. Other distinctive features of the algorithm are that it is free from local minima, the obstacles can have any shape, low cost sensors can be used to detect obstacles and an appropriate balance is achieved between the use of the global and the local approaches for collision avoidance. Our present work is a refinement of this strategy that allows for an automatic real time adaptation of the algorithm's parameters. Now, the algorithm's functioning requires only that the minimum distance at which the AGV can approach an obstacle (i.e. the closest it can get to any obstacle) is defined a priori. Aspects of the real implementation of the algorithm are also discussed.
\end{abstract}

Index Terms - Obstacle avoidance, potential fields, adaptive trajectory planning, autonomous guided vehicle.

\section{INTRODUCTION}

This paper discusses new developments on the application of the Blind Person Strategy for Obstacle Avoidance using the Potential Fields algorithm proposed by Lopes [1] for the navigation of the CONTROLAB AGV within a set of rooms in a building. As discussed in [1], [2], [3], the CONTROLAB AGV indoor navigation problem has been set and solved as follows. The CONTROLAB AGV is a tricycle vehicle which displays circular symmetry and has a set of infrared sensors on the lower end of its front part. These sensors act as an electronic stick, being able to detect obstacles as the AGV moves. The AGV is initially provided with the floor plan of the building, but has no knowledge of any obstacles which might be present within this floor plan.

${ }^{*}$ This work is supported by FINEP (Research and Projects Financing)
The AGV may plan its trajectory from any starting position to any destination within this building. The planning of this trajectory is divided in two phases. In the first phase, the global path planning is tackled: a set of doors which the AGV has to cross in order to go from its starting position to the requested end position, moving through the lowest cost trajectory, is determined. This problem is casted as a shortest-path problem in a directed graph and solved, by well-known algorithms, before the AGV starts moving.

In the second phase of the trajectory planning, the precise trajectory to be followed by the AGV within a room is defined by a global navigation function related to this particular room. This function is a harmonic potential function calculated on a grid that is laid out in the room area. As is done in most applications of the potential field strategy for robot navigation, our AGV moves in the opposite direction to the gradient of the available global navigation function. During the AGV's movement, the global navigation function is modified to incorporate information about detected obstacles. This adaptation of the global navigation function is performed by the addition of two grids: the grid of the present navigation function multiplied by a parameter and the grid of the potential function associated with the detected obstacle point multiplied by another parameter. These two parameters used to be determined by heuristic methods at each interaction of the algorithm and they had to be set in such a way as to guaranty that the addition of the potential function associated with a newly detected obstacle point would neither provoke too much growth in the potential field inside the room (which would cause the AGV to be pushed into the walls) nor too little growth in the potential field near the detected obstacle (which would prevent the AGV from avoiding it). In the present work, the equations of our previous work [1] are rewritten in order to replace the old parameters by new ones that have a clear physical signification. These parameters are now either determined before the AGV starts moving or mathematically determined in real-time whenever a new obstacle is detected. The first new parameter used is the minimum radius of a detected obstacle point $\left(r_{m}\right)$. This parameter expresses the minimum distance at which the AGV is allowed to approach an obstacle. The minimum radius needs to be defined only once since this parameter is only dependent upon the dimensions of the robot and the 
level of obstacle collision risk chosen. Another parameter introduced is $\lambda_{n}$, which establishes the level of repulsion of the $n^{t h}$ obstacle point. This parameter must be such that it prevents the robot from coming closer than a distance $r_{m}$ to the obstacle point. The parameter $\lambda_{n}$ is calculated as a function of $r_{m}$ and of the $n^{\text {th }}$ obstacle point's position. A final parameter introduced, $r_{M}$, gives rise to an interesting skill acquired by the robot when commanded by our algorithm. The AGV is now able to discard any information which can deviate it towards the room walls by neglecting obstacle points which are further away from it than a distance $r_{M}$. On detecting such obstacle points, the AGV decreases the length of its stick so as to only "see" obstacles close enough to need to be avoided. If however, no obstacle points are detected with this stick length, the stick is gradually increased again. On the other hand, when a detected obstacle point is within the maximum and minimum radii, it is assigned a repulsive charge. In this case, the stick's length is maintained.

The use of virtual potential fields for obstacle avoidance was introduced by Khatib [4]. Potential field methods and other global and local techniques used in robot motion planning have been treated in detail by Latombe [5]. The importance of harmonic potential functions in the generation of artificial potential fields has been stressed by Kim and Khosla [6], [7]. In their influential work, Koren and Borenstein [8] spotted the difficulties of the use of the potential field method as being: trap situations due to local minima, incapacity of finding trajectories between closely spaced obstacles, and the oscillatory behavior of trajectories that follow walls and narrow passages. The proposed methods which followed Koren's and Borenstein's work, such as the Vector Field Histogram method [9],[10] and the Dynamic Window method [11], overcame these difficulties through solutions based on elaborated calculation of the vector field and optimization procedures for choosing the velocity and the direction of the robot at each step. As will be shown, our solution for the CONTROLAB AGV indoor navigation problem also overcame the mentioned difficulties. Our method uses harmonic vector fields to take advantage of the availability of a floor plan to allow much of the burden of field calculation to be dealt with before the AGV starts moving. This leaves only easy vector field update operations and fifth-order polynomial trajectory interpolations [3] to be done while the robot is moving.

In Section 2 of this work, the global navigation function and its real-time adaptation are discussed and the real-time calculation of the parameters is explained. In Section 3, results of simulation experiments are presented. Finally, in Section 4, the main conclusions are formulated.

\section{The Global Navigation Function ANd its ADAPTATION}

\section{A. The Potential Field results used in this work}

A complete account of the potential field results used in this work can be found in Epstein [12]. A set $\Gamma \subset \Re^{n}$ is a domain if it is open and connected. A function $\mathrm{f}$ defined in a domain $\Gamma$ and belonging to the class $C^{2}(\Gamma)$ is said to be harmonic in $\Gamma$ if $\nabla^{2} f=0$, where $\nabla^{2}=\partial^{2} / \partial x_{1}^{2}+\ldots+$ $\partial^{2} / \partial \mathbf{x}_{n}^{2}$. Harmonic functions have the following properties:

1) A linear combination of harmonic functions is harmonic;

2) A harmonic function's minimum or maximum within a domain is attained at the boundaries of the domain;

3) A harmonic function remains harmonic if an affine transformation is applied to its variables.

\section{B. Construction of the global navigation function for a room}

As said before, the global navigation function for a room is initially determined by the information available before the AGV starts moving. This information consists of: the AGV's position within the room; the length (d) and the width (b) of the room (which is supposed to be a rectangle) and the position of the door $\left(d, y_{0}\right)$ to be reached (see Fig 3). This information indicates that the initial global navigation function $F_{0}$ can be defined as a solution of the following Dirichlet problem for the Laplace equation:

$$
\begin{gathered}
\frac{\partial^{2} u}{\partial x^{2}}+\frac{\partial^{2} u}{\partial y^{2}}=0 \\
u(x, y)=0, \text { for }(x=0 \text { and } 0 \leq y \leq b) \text { or } \\
(y=0 \text { and } 0 \leq x \leq d) \text { or }(y=b \text { and } 0 \leq x \leq d) \\
u(d, y)=u_{0}, \text { for } 0<y_{0} \leq y \leq y_{0}+\Delta y,
\end{gathered}
$$

where $u_{0}<0$;

$$
u(d, y)=0 \text {, for }\left(0<y<y_{0}\right) \text { or }\left(y_{0}+\Delta y<y \leq b\right) .
$$

The domain in which this problem is defined is noted $\Gamma_{0}$ and represents the room area.

The problem above has a closed solution which is given by:

$$
u(x, y)=\sum_{k=1}^{\infty} E_{k} \sinh (k \Pi x / b) \sin (k \Pi y / b),
$$

where:

$$
E_{k}=\frac{2 u_{0}\left(\cos \left(k \Pi y_{0} / b\right)-\cos \left(k \Pi\left(y_{0}+\Delta y\right) / b\right)\right)}{\Pi k(\sinh (k \Pi d / b))}
$$

This solution is evaluated in the grid points before the AGV starts moving. The grid values of a radial harmonic potential function $\phi$, centered at the point $(0,0)$, are also calculated before the $\mathrm{AGV}$ starts moving.This function is given by:

$$
\phi(x, y)=(-1 / 2 \Pi) \log r, \text { where } r=\sqrt{x^{2}+y^{2}} .
$$

The AGV always moves in the opposite direction to the gradient of the available global navigation function 
and, when the electronic stick detects an obstacle point, a new global potential function for the room is generated by adding to the old global potential function, a function obtained by a transformation of $\phi$. This can be written as follows.

If $F_{n}$ is the global potential function when the $n^{t h}$ point is detected, then the new global potential function is:

$$
F_{n+1}(x, y)=F_{n}(x, y)+T_{n+1}(\phi)(x, y)
$$

where the operators $T_{n+1}$ transform $\phi$ into a new harmonic function in the domain $\Gamma_{n+1} \subset \Gamma_{n} \subset \ldots \subset \Gamma_{0}$, as will be discussed next.

\section{The operator $T_{n}$}

The operator $T_{n}$ is defined by:

$$
\begin{gathered}
T_{n+1}(\phi)(x, y)=\lambda_{n+1}\left[\phi\left(x-x_{n+1}, y-y_{n+1}\right)-\right. \\
\phi\left(\tilde{x}_{n+1}-x_{n+1}, \tilde{y}_{n+1}-y_{n+1}\right), \forall(x, y) \in \Gamma_{n+1}
\end{gathered}
$$

where

1) $\left(x_{n+1}, y_{n+1}\right)$ is the grid point nearest to the $(n+1)^{t h}$ obstacle point detected;

2) $\Gamma_{n+1}=\Gamma_{n} /\left\{\left(x_{n+1}, y_{n+1}\right)\right\}$;

3) $\left(\tilde{x}_{n+1}, \tilde{y}_{n+1}\right)$ is the farthest corner of the room from the $(n+1)^{t h}$ detected obstacle point;

4) $\lambda_{n+1}$ is a positive real number.

Further explanation is needed for an easier understanding of the form of the proposed operator $T_{n}$ :

1) Point $\left(x_{n+1}, y_{n+1}\right)$ is chosen as the symmetry center for function $T_{n+1}(\phi)$, which represents the repulsion caused by the presence of the $(n+1)^{t h}$ detected obstacle point;

2) $\phi\left(\tilde{x}_{n+1}-x_{n+1}, \tilde{y}_{n+1}-y_{n+1}\right)=C_{n+1}$ is a constant which guarantees that

$$
T_{n+1}(\phi)(x, y) \geq 0
$$

and

$$
u_{0} \leq F_{n}(x, y) \leq F_{n+1}(x, y)
$$

for all $(x, y) \in \Gamma_{n+1}$;

3) Due to the first and the third previously mentioned properties of harmonic functions, $F_{n}$ is a harmonic function in $\Gamma_{n}$, for all $\mathrm{n}$;

4) the value of $\lambda_{n+1}$ controls the level of repulsion of the $(n+1)^{t h}$ obstacle point detected, which grows with $\lambda_{n+1}$. From (1) and (2) one can conclude that the proposed algorithm is completely determined by the definition of $\lambda_{n}$, for each $\mathrm{n}$.

\section{Determination of $\lambda_{n}$}

Let $\left(\breve{x}_{n+1}, \breve{y}_{n+1}\right)$ be the position of the symmetric center of the AGV at the time when the $(n+1)^{t h}$ obstacle point is detected, the vector $(w, z)$ be the unitary vector in the direction of vector $\left(\breve{x}_{n+1}-x_{n+1}, \breve{y}_{n+1}-y_{n+1}\right)$ and

$$
P(t)=F_{n+1}\left(x_{n+1}+t w, y_{n+1}+t z\right) .
$$

Function $\mathrm{P}$ can also be written as:

$P(t)=F_{n}\left(x_{n+1}+t w, y_{n+1}+t z\right)-\frac{\lambda_{n+1}}{2 \Pi} \log (t)+\lambda_{n+1} C_{n+1}$ and its derivative is:

$\frac{d}{d t} P(t)=<\nabla F_{n}\left(x_{n+1}+t w, y_{n+1}+t z\right),(w, z)>-\frac{\lambda_{n+1}}{2 \Pi t}$

Consider $t_{M}>0$ such that $\left(x_{n+1}+t_{M} w, y_{n+1}+t_{M} z\right) \in$ $\partial \Gamma_{0}$.

Except in very especial cases, when the straight line from the point $\left(x_{n+1}, y_{n+1}\right)$ in the direction of vector $(w, z)$ reach an obstacle point that have already been detected, function

$$
t \mapsto<\nabla F_{n}\left(x_{n+1}+t w, y_{n+1}+t z\right),(w, z)>
$$

is bounded by the above, is positive and decreasing in $\left(0, t_{M}\right)$.

As

$$
\lim _{t \rightarrow 0} \frac{\lambda_{n+1}}{2 \Pi t}=+\infty
$$

then $\exists t_{1} \in(0,+\infty)$, such that,

$$
\frac{d}{d t} P(t)<0, \text { for } t \in\left(0, t_{1}\right) \text { and } \frac{d}{d t} P\left(t_{1}\right)=0 .
$$

Therefore the minimum radius of a detected obstacle point can be defined as the strictly positive minimal number $r_{m}$ such that

$$
P^{\prime}\left(r_{m}\right)=0 \text {. }
$$

By this definition, the minimum radius $r_{m}$ of a detected obstacle point is a local minimum of function $\mathrm{P}$ and is the maximum distance which the $\mathrm{AGV}$ can approach the obstacle point detected when it moves from its current position $\left(\breve{x}_{n+1}, \breve{y}_{n+1}\right)$ in the direction $-(w, z)$. The definition of this value depends on the application or, in other words, how close to the obstacle the AGV can risk to be. When $r_{m}$ is fixed, the value of $\lambda_{n+1}$ can be calculated as follows:

$$
\begin{gathered}
0=P^{\prime}\left(r_{m}\right) \\
=\left.\left\langle\nabla F_{n}\left(x_{n+1}+t w, y_{n+1}+t z\right),(w, z)\right\rangle\right|_{t=r_{m}} \\
+\left.\frac{d}{d t} T_{n+1}(\phi)\left(x_{n+1}+t w, y_{n+1}+t z\right)\right|_{t=r_{m}} \\
=\left\langle\nabla F_{n}\left(x_{n+1}+r_{m} w, y_{n+1}+r_{m} z\right),(w, z)\right\rangle \\
+\left.\lambda_{n+1}\left[\frac{d}{d t} \phi(t w, t z)\right]\right|_{t=r_{m}} .
\end{gathered}
$$




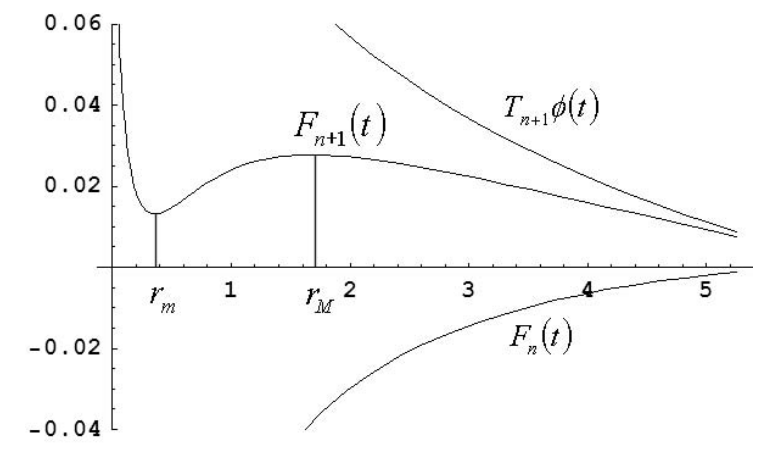

Fig. 1. The Global Navigation Function $F_{n+1}(t)$ is adapted by using $F_{n}(t)$ and $T_{n+1}(\phi)(t)$, as described in equation 1 .

Then

$$
\begin{aligned}
& \lambda_{n+1}=-\frac{\left\langle\nabla F_{n}\left(x_{n+1}+r_{m} w, y_{n+1}+r_{m} z\right),(w, z)\right\rangle}{\left.\left[\frac{d}{d t} \phi(t w, t z)\right]\right|_{t=r_{m}}} \\
& =2 \Pi r_{m}\left\langle\nabla F_{n}\left(x_{n+1}+r_{m} w, y_{n+1}+r_{m} z\right),(w, z)\right\rangle .
\end{aligned}
$$

\section{E. Limitation on the size of the electronic stick}

It is also possible to define a maximum radius of a detected obstacle point as the number $r_{M}$ such that

$$
r_{M}=\min \left(\left\{t \in\left(r_{m}, t_{M}\right) \mid P^{\prime}(t)=0\right\} \bigcup\left\{t_{M}\right\}\right)
$$

Often, $r_{M}$ is a local maximum of function $\mathrm{P}$. This means that, if the symmetric center of the $\operatorname{AGV}\left(\breve{x}_{n+1}, \breve{y}_{n+1}\right)$ at the time when the $(n+1)^{t h}$ obstacle point is detected is far from $\left(x_{n+1}+r_{M} w, y_{n+1}+r_{M} z\right)$ (i.e. $r_{M}<\|$ $\left.\left(\breve{x}_{n+1}-x_{n+1}, \breve{y}_{n+1}-y_{n+1}\right) \|\right)$ then the AGV tends to go in the direction of the wall. This observation indicates that a limitation in the size of the stick to be used by the $\mathrm{AGV}$ is necessary in order to avoid that the AGV hits the wall. Fig.1 e Fig.2 summarize the previous discussion.

When a new global navigation function replaces the old one, a discontinuity on the direction of the trajectory of the AGV is introduced. This discontinuity is more severe when the AGV is close to the obstacle. The constraints of the AGV's dynamics indicate that a long stick is preferable to a short one. However, our calculation shows that the stick's size can't be longer than the maximum radius $r_{M}$ and that this last number varies with the shape of the global potential function in use and with the definition of the minimum radius $r_{m}$. When the stick size is longer than $r_{M}$ the obstacle point must be ignored and the stick's size decreased. Each time the potential field compels an important change in the AGV movement direction the stick return to its initial size.

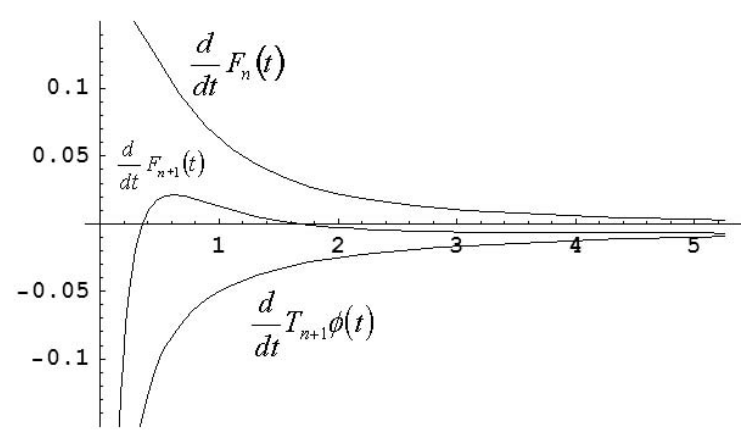

Fig. 2. The derivatives of $F_{n}, T_{n+1}(\phi)$ and $F_{n+1}$.

\section{Simulated EXPERIMENTS}

The simulated experiments have been performed on a Pentium@233 MHz computer and the algorithm has been implemented in C (GCC on MSDOS). In the current implementation, the algorithm takes $7.32 \mathrm{~ms}$ to adapt the Global Navigation Function for a grid of (46 X 59) cells. The experiments have been carried out considering a rectangular room with a single door and a set of obstacles which were placed in such a way as to cause interesting potential field configurations to arise. Initially, the lowest potential value was placed at the $\operatorname{door}\left(u_{0}=-1\right)$ and the highest potential value, set as 0 , was attributed to the walls. Fig. 3 shows the AGV, within the room, following the trajectory dictated by the initial potential field before any obstacles have been detected by the stick. The initial potential field is calculated before the AGV starts moving and its values are mapped on the grid positions. The same is done, also before robot motion, for values of a potential function $\phi$ that represents the charge potential field to be associated with each obstacle point detected. Each time an obstacle point is detected, the updated potential field causes a strong repulsion near that obstacle. As we move along a straight line direction from the obstacle to the $\mathrm{AGV}$, the repulsion firstly diminishes, reaching zero at the minimum radius and then shifts direction, changing from a repulsive to an attractive force. This attraction reaches 0 at the maximum radius and then changes direction again to become a repulsion as we move towards the wall (see Fig 1 and Fig 2). Fig.4 shows equipotential lines of the adapted field generated when the electronic stick detects an obstacle. The arrows shown in all figures point to the decreasing potentials. It can be seen in Fig 4 that a saddle point is created between the obstacle and the AGV at the minimum radius, causing the $\mathrm{AGV}$ to avoid the obstacle. The complete AGV real-time movement is shown in Fig.5. As discussed in Section 2, the size of the electronic stick must be smaller than the maximum radius in order to keep the AGV in front of the potential "wave" that leads it to the door. Fig. 4 shows the resulting field when the stick used is of the correct size. It can be seen in Fig.6 that the AGV is being pushed against the wall as it is located behind the potential "wave". The simulation of a 


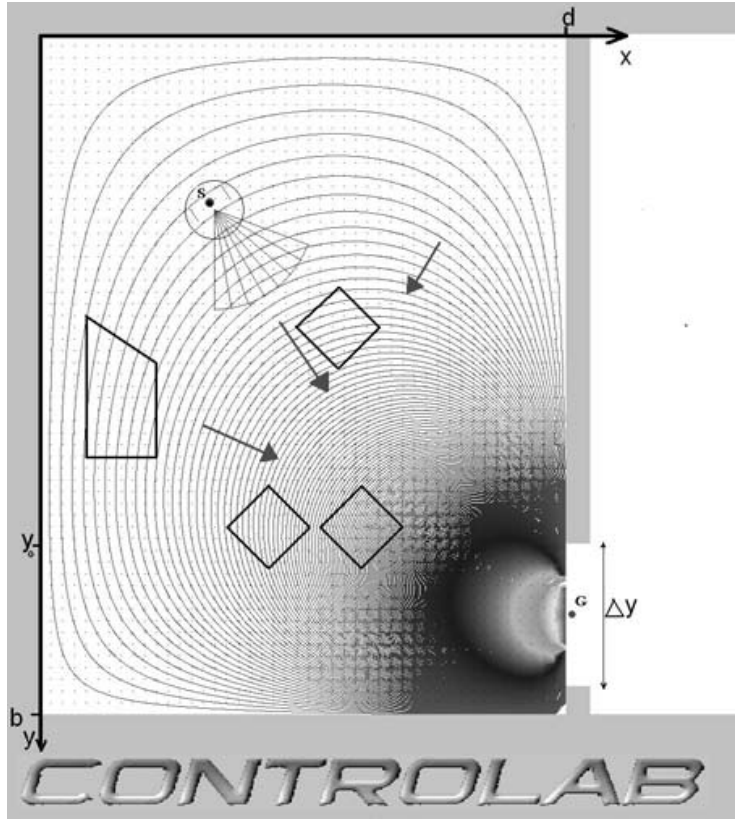

Fig. 3. Initial Room Potential Field.

challenging situation where the AGV has to follow long walls and narrow passages is displayed in Fig.7 and Fig.8.

The strong attraction of the door and the adaptive nature of the stick's range enable the correct placement of charges that conduct the AGV to the door. The real-time experiments with the AGV show that the time taken for the controller to control the present set point velocities and to generate the next feasible ones is $5.93 \mathrm{~ms}$. Adding this time to the $7.32 \mathrm{~ms}$ needed to update the field, we can conclude that the algorithm can control robots demanding a control interval as short as $15 \mathrm{~ms}$.

\section{CONCLUSION}

We have solved the problem of automatically determining, in real-time, the values of the parameters of the proposed algorithm. When the size of the minimum radius $r_{m}$, which depends on safety precautions, is chosen, the algorithm is able to automatically generate a sequence of potential functions $F_{n}$ and a sequence of parameters $\lambda_{n}$ which enable the AGV to reach the door within any reasonable situation. A test must be done each time a new obstacle is detected in order to verify if the stick size used is greater than the maximum radius $r_{M}$. If it so is, the stick's length is reduced. When the stick's size is constantly small, implying a rough AGV trajectory, its necessary to smoothen out the trajectory curve (as discussed in [3]) so as to satisfy the dynamic constraints of the robot. The simulation presented showed the effectiveness of the algorithm in a challenging situation and the successful results of our study.

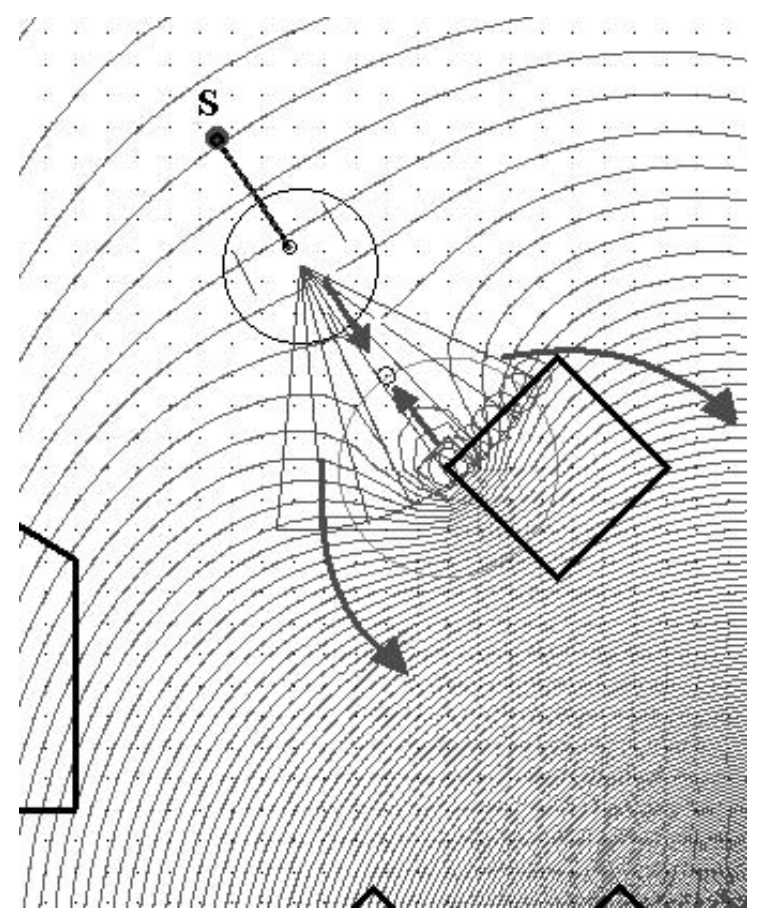

Fig. 4. Equipotential lines of the adapted field. Here, the robot's stick is shorter than the maximum radius $r_{M}$

\section{REFERENCES}

[1] E.P. Lopes et al,"Application of a Blind Person Strategy for Obstacle Avoidance with the use of Potential Fields",Proc.IEEE I CRA,Seoul,Korea,pp. 2911-2916,May 2001.

[2] E.P.L.Aude et al,"CONTROLAB MUFA:A Multi-Level Fusion Architecture for Intelligent Navigation of Tele-robot",Proc.IEEE ICRA,Detroit,USA,pp.465-472,May 1999.

[3] E.P.L.Aude et al,'Real-Time Obstacle Avoidance Performed by an Autonomous Vehicle Throughout a Smooth Trajectory Using an Electronic Stick",Proc. IEEE IROS,Las Vegas,Nevada,pp.898905, October 2003.

[4] O. Khatib,"Real-time obstacle avoidance for manipulator and mobile robots",Proc. of the IEEE ICRA,St.Louis,Missouri,USA,pages 500505,1985

[5] J.C.Latombe,'Robot Motion Planning", Kluwer Academic Publishers, 1991.

[6] J.Kim,P.Khosla,'Real-Time Obstacle Avoidance using Harmonic Potential Functions",Proc.IEEE ICRA, Sacramento,USA,pp.790796,April1991.

[7] J.Kim,P.Khosla,'Real-Time Obstacle Avoidance using Harmonic Potential Functions",IEEE Transaction on Robotics and Automation,June 1992.

[8] Y.Koren,J.Borenstein,'Potential Field Methods and Their Inherent Limitations for Mobile Robot Navigation",Proc. of the IEEE ICRA, Sacramento, California,pages 1398-1404, April 1991.

[9] I.Ulrich, J.Borenstein,'VFH+:Reliable Obstacle Avoidance for Fast Mobile Robots",Proc. of the IEEE ICRA, Leuven, Belgium, pages 1572-1577, May 1998.

[10] I.Ulrich, J.Borenstein,'VFH*:Local Obstacle Avoidance with LookAhead Verification",Proc. of the IEEE ICRA, San Francisco, California, USA, pages 2505-2511, April 2000.

[11] D.Fox, W.Burgard,S.Thrun,'The Dynamic Window Approach to Collision Avoidance",IEEE Robotics and Automation Magazine, pages 23-33, March 1997.

[12] B.Epstein,'Partial Differential Equations:An Introduction",McGrawHill Book Company, 1962. 


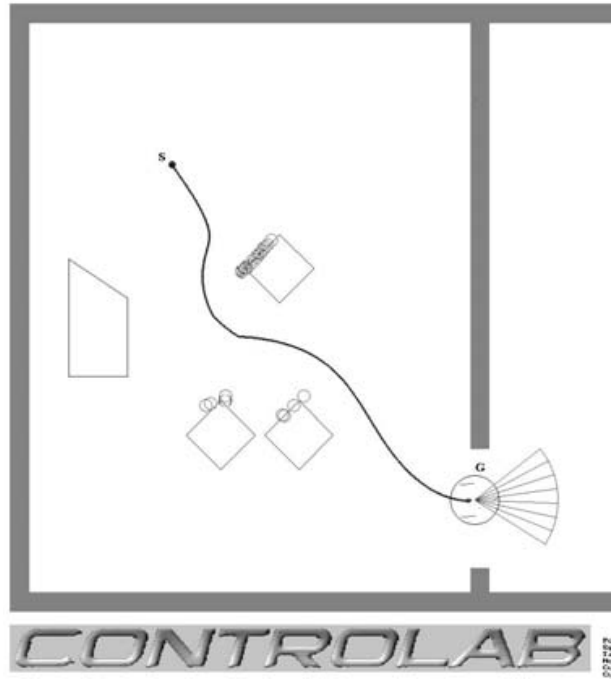

Fig. 5. The AGV real-time movement.

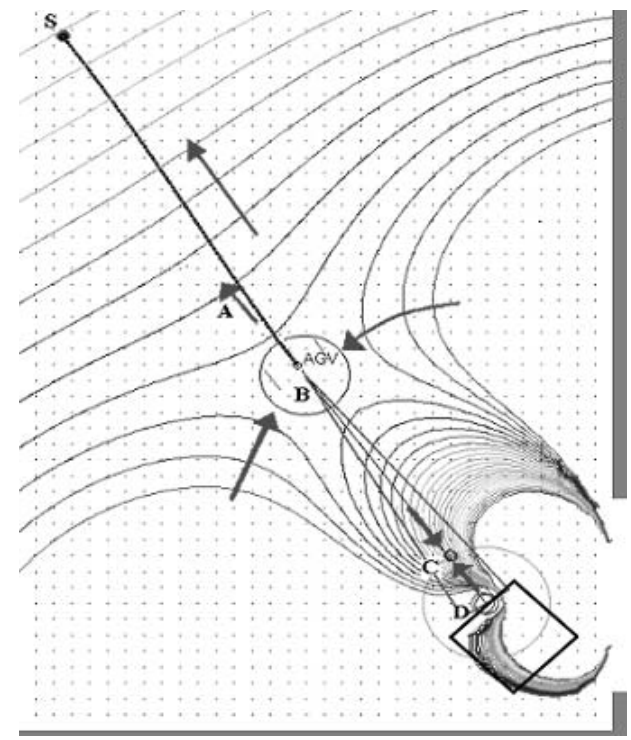

Fig. 6. Here, the AGV is slightly behind the potential wave. i.e. the stick used is longer than the maximum radius $r_{M}$

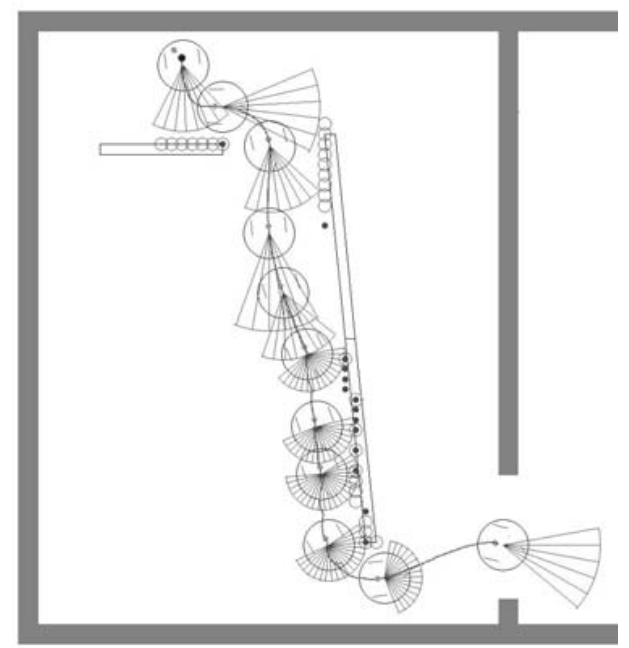

Fig. 7. The AGV following long walls and crossing narrow passages with its adaptive stick.

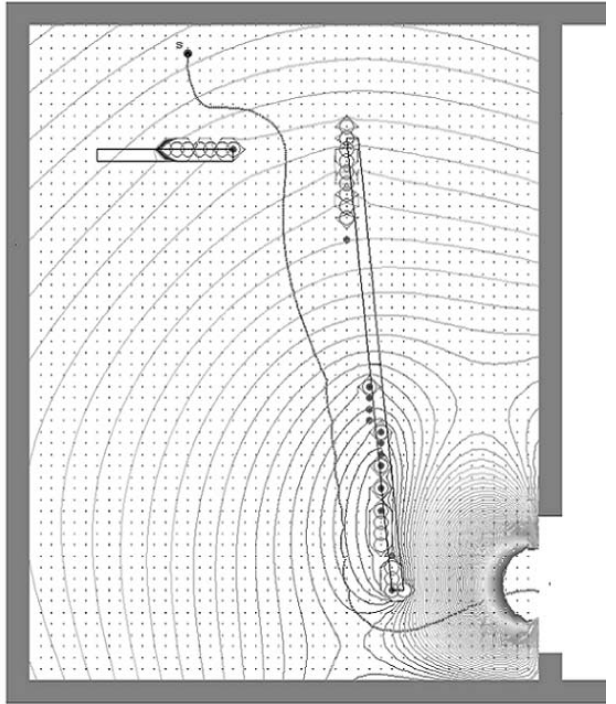

Fig. 8. The Final Field and the AGV trajectory produced by the motion in Fig 7. 